http://dx.doi.org/10.35381/racji.v5i3.1091

\title{
Violencia Intrafamiliar durante el período de confinamiento por pandemia COVID-19
}

\section{Intrafamily violence during the period of confinement due to COVID-19 pandemic}

\author{
Laura Alicia Colcha-Ramos \\ ur.lauracolcha@uniandes.edu.ec \\ Universidad Regional Autónoma de los Andes, Riobamba \\ Ecuador \\ https://orcid.org/0000-0002-4805-7271 \\ Letty Elizabeth Córdova-Requena \\ dr.lettyecr73@uniandes.edu.ec \\ Universidad Regional Autónoma de los Andes, Riobamba \\ Ecuador \\ Erika Guadalupe Salguero-Abarca \\ dr.erikagsa46@uniandes.edu.ec \\ Universidad Regional Autónoma de los Andes, Riobamba \\ Ecuador
}

Recibido: 31 de octubre de 2020

Revisado: 10 de octubre de 2020

Aprobado: 05 de diciembre de 2020

Publicado: 10 de diciembre de 2020 


\title{
RESUMEN
}

La investigación tiene por objetivo principal analizar la violencia Intrafamiliar durante el período de confinamiento por pandemia covid-19 en la ciudad de Riobamba - Ecuador. De carácter metodológico descriptivo con diseño bibliográfico no experimental. Se identificaron un total de 1695 atenciones relacionadas con casos de violencia intra familiar. Se puede evidenciar que la pandemia por COVID-19, ha generado que varias familias pierdan el trabajo, dando así a que los estudiantes no puedan acceder a la educación porque no cuentan con los medios suficientes, debido a que sus padres no gozan de un trabajo estable y los problemas en el hogar son diariamente siendo más propensos a que sufran de violencia intrafamiliar.

Descriptores: Violencia doméstica; problema social; derecho civil. (Palabras tomadas del Tesauro UNESCO).

\begin{abstract}
The main objective of the research is to analyze intrafamily violence during the period of confinement due to the covid-19 pandemic in the city of Riobamba - Ecuador. Descriptive methodological in nature with non-experimental bibliographic design. A total of 1695 attentions related to cases of intra-family violence were identified. It can be seen that the COVID-19 pandemic has caused several families to lose their jobs, thus preventing students from accessing education because they do not have sufficient means, because their parents do not have a job. stable and problems at home are daily being more likely to suffer from domestic violence.
\end{abstract}

Descriptors: Domestic violence; social problems; civil law. (Words taken from the UNESCO Thesaurus). 
lustitia Socialis. Revista Arbitrada de Ciencias Jurídicas.

Año V. Vol. V. №3. Edición Especial. 2020-III:

Universidad Regional Autónoma de los Andes

Hecho el depósito de Ley: FA2016000064

ISSN: 2542-3371

FUNDACIÓN KOINONIA (F.K). Santa Ana de Coro, Venezuela

Laura Alicia Colcha-Ramos; Letty Elizabeth Córdova-Requena; Erika Guadalupe Salguero-Abarca

\section{INTRODUCCIÓN}

En relación a la violencia en el interior de la familiar, se entiende como la violencia que se vive dentro del hogar tiene diferentes índoles según el país porque depende de los factores económicos y sociales. En este sentido, (Cisneros-Trujillo, 2006), define la violencia intrafamiliar como "los niveles de participación y responsabilidad de los varones y mujeres mantienen en la producción y reproducción de la violencia intrafamiliar y los significados y valoraciones que nuestra sociedad les asigna a los conceptos de violencia y familia", lo cual se evidencia como un problema social causada por la interrelación de factores culturales, económicos y psicológicos.

Por lo tanto, la violencia intrafamiliar se comprende como el abuso que ejercen unos miembros de la familia sobre otros. Puede ser física, sexual o psicológica, y causar daño, sufrimiento físico, sexual y psicológico, esto quiere decir que la violencia intrafamiliar involucra, insultos, gritos, golpes, daño psicológico sexual y muerte a cualquier miembro de la familia (Mayor-Walton \& Salazar-Pérez, 2019). Por otro lado, el Código Orgánico Integral Penal, COIP, en su artículo 155, define a la violencia intrafamiliar como "Toda acción que consista en maltrato, físico, psicológico o sexual ejecutado por un miembro de la familia en contra de la mujer o demás integrantes del núcleo familiar", siendo esto compaginado con la visión internacional de que podrían desarrollarse diversos tipos de violencia dentro del hogar.

Prosiguiendo con lo planteado, (Romero-Bello \& González-Blanco, 2017), destacan en su investigación, una frecuencia de $43,4 \%$ de violencia familiar en orden psicológico, física y sexual, expresado por las mujeres que acudían a su consulta ginecológica, lo cual, evidencia que la mujer es una de las integrantes de mayor afección por algún tipo de violencia en el seno del hogar, generalmente producida por el hombre caracterizado por estereotipos como el machismo, así como inmerso en vicios como el alcohol o drogas, lo cual concuerda con (Puente-Martínez, et al., 2016).

Siendo una tendencia de violencia que aparentemente se mantiene, en contraste con el informe de la Organización Panameriana de la salud del año 2002, donde se destaca que una de cada tres mujeres son afectas por violencia intrafamiliar en las Américas, convirtiéndose en un problema complejo, acontecimiento que se lo puede palpar con 
mayor frecuencia en las comunidades rurales pobres con falta de servicios y lejanía y puede ser causa por factores como el alcoholismo, el desempleo o la decencia económica convirtiéndose en algo natural y común (Organización Panameriana de la salud, 2002).

Según (De-Alencar-Rodrígues \& Cantera, 2013), la violencia de género incluye todo tipo de violencia contra la mujer basada en la pertenencia del género femenino, que trasciende los límites de las relaciones familiares y afectivas. Se da una violación de los derechos humanos. Además, está clasificado como un problema grave de salud física y mental y ha alcanzado proporciones epidémicas, afectando al $30 \%$ de las mujeres en todo el mundo.

Según El Instituto Nacional de Estadística y Censos señala que el $65 \%$ de las mujeres en Ecuador han experimentado violencia de género, de las cuales el $45 \%$ fueron cometidas por sus parejas, la violencia intrafamiliar ha aumentado debido a las tensiones y conflictos, las medidas de cuarentena domiciliaria son necesarias para prevenir la propagación del COVID-19, y muchas mujeres se ven obligadas a quedarse en casa con los agresores, poniendo en riesgo sus vidas. (INEC, 2020)

Las mujeres ecuatorianas son quienes han representado más trabajo debido al cierre de las escuelas, situación que se tomó como prevención ante la pandemia, a su vez tienen responsabilidades con sus familiares, niñas y niños que deben atenderlos y estar al pendiente de su salud lo que resulta una sobrecarga de las labores dentro del hogar, toda esta presión de trabajo puede generar violencia intrafamiliar (INEC, 2020).

La incidencia de violencia intrafamiliar se ha incrementado durante la pandemia, esta condición en el hogar no ha permitido el buen desarrollo de los niños, niñas y adolescentes: "El incremento de la violencia durante la crisis muestra que sus causas estructurales y factores culturales no han sido erradicados y que resultan débiles los mecanismos de denuncia, atención y respuesta" (Montero-Medina, et al., 2020).

El aislamiento implica no estar en contacto con la sociedad esto dificulta que las mujeres mantengan el apoyo de familiares, vecinos, aquí la mujer tendrá que estar aislada con el agresor, es complicado buscar ayuda debido a la crisis sanitaria, la mayoría de los servicios están dirigidos a personas sospechosas y con COVID-19, dejando a un lado a 
los afectados por violencia doméstica o de género (López-Hernández \& Rubio-Amores, 2020).

Para la Organización Mundial de la Salud, las condiciones y formas de vivencia de los miembros ecuatorianos no son los adecuados en el ámbito de la salud pública, como es en el caso de la pandemia de COVID-19 donde se simboliza una desigualdad social y económica donde se pueden determinar varios grupos entre ellos las personas expuestas al virus y las más vulnerables. (Organización Mundial de la Salud, 2020) Las poblaciones ecuatorianas en el ámbito laboral no cuentan con un empleo estable para solventar su economía, su condición de salud ha producido que se enfrenten acatar las medidas tomadas en el periodo de pandemia, dando como resultado muchas dificultades para tener buenos ingresos de subsistencia. (Guerra \& Beltrán, 2020).

EI ECU911 a determinado un total de 8.196 llamadas que se registraron durante el mes de marzo 2020 relacionadas a la violencia contra la mujer o miembros familiares: "En el período del 1 al 16 de marzo y 2.938 llamadas de emergencias recibidas entre el 17 al 31 de marzo, en el decreto de excepción por la emergencia sanitaria COVID-19; esto es 234 llamadas de auxilio al día" (Servicio Integrado de Seguridad ECU 911, 2020)

Los casos de violencia intrafamiliar ponen en riesgo y elevan la vulneración de las víctimas debido a que el aislamiento también puede dar como resultado que le agresor presione a los perjudicados y los induzca a no presentar las quejas, enfrentándose a una cruel realidad, don los más afectados son los menores de edad es así que se reseña que "desde que se habilitó la herramienta de denuncia en línea, en la página web de Fiscalía, se ingresaron 2.443 formularios. Luego del análisis jurídico correspondiente, se han procesado 1.742 denuncias de presuntos delitos de violencia de género o intrafamiliar" (Metro Ecuador, 2020), por consiguiente, se ha apostado en generar mecanismos para reducir la brecha de la violencia familiar, especialmente la enfocada contra la mujer.

El $64 \%$ de mujeres en Ecuador han sufrido algún tipo de violencia basada en género, el $30 \%$ dentro de sus hogares y en un $43 \%$ de los casos el perpetrador es el compañero. Además, la probabilidad de sufrir violencia cuando el compañero está en casa o en desempleo aumenta en un 28 \%, (Chacón, 2020). 
Welland (2017) menciona que es un deber y responsabilidad de cada uno de ayudar y prevenir que más familias sufran de violencia intrafamiliar que debemos ayudar a la víctima cuando nos pide ayuda ya que puede estar en juego su vida, apoyar no significa decirle a la persona agredida una y otra vez que debería abandonar a su pareja, sino hay que ofrecerle confianza que sienta que no está sola

Por otro lado, (Segura-Vera \& Stranieri, 2016) indica que los servicios de salud pueden ser muy importante en la atención de familias que sufren de violencia, deben pedir ayuda profesional aquí en el Ecuador existen instituciones para ayudarte a las personas que son víctimas, por cuanto las personas profesionales les acompañarán en todo el proceso de salida de la violencia aquí hablan con el agresor, si lo podemos hacer, sin poner en riesgo la propia vida o la de la pareja.

En razón de lo planteado, la investigación realiza una revisión bibliográfica sobre los aspectos fundamentales acerca del incremento de violencia intrafamiliar donde las víctimas son las mujeres durante el período de confinamiento por la pandemia de COVID19 en la ciudad de Riobamba, analizando la situación en la que viven utilizando la normativa vigente donde se señalan las consecuencias que sufren el género femenino, cuantificando el número de casos existentes de violencia intrafamiliar durante el período del mes de marzo y junio del 2020, identificando los diferentes tipos de violencia intrafamiliar que ocasionan a la víctima y por último utilizando trípticos sobre los derechos que han sido vulnerados como medios informativos para concientizar a los miembros de la población.

En este sentido, se tiene por objetivo principal analizar la violencia Intrafamiliar durante el período de confinamiento por pandemia covid-19 en la ciudad de Riobamba - Ecuador.

\section{MÉTODO}

La investigación es de carácter metodológico descriptivo con diseño bibliográfico no experimental, abordándose una población de documentos que fueron analizados mediante el método analítico - sintético con la finalidad de conocer los elementos que constituían un aporte para configurar resultados y conclusiones sobre el tema abordado, aplicándose la técnica de análisis de contenido para escrutar la información 
Laura Alicia Colcha-Ramos; Letty Elizabeth Córdova-Requena; Erika Guadalupe Salguero-Abarca

seleccionada.

\section{RESULTADOS}

Se identificaron un total de 1695 atenciones relacionadas con casos de violencia intra familiar, se priorizaron estudios basados en el análisis de las necesidades que tienen las mujeres siendo víctimas de maltrato intrafamiliar, determinando las consecuencias que se ocasionó, se analizó los estudios acerca de las perspectivas de los diferentes autores acerca de la violencia intrafamiliar, profundizando los datos haciendo un énfasis en las barreras y las dificultades para dar una propuesta de mejora.

Se resalta por parte del Ministerio de Agricultura y Agricultura (2019), el rol de las mujeres como ente productivo de la nación, lo cual involucra que las mujeres son el principal pilar fundamental en el hogar, porque están a cargo de todas las actividades asumiendo el cuidado y el desarrollo de los miembros de su familia, en el caso de pandemia producido por COVID-19 se ha tomado medidas necesarias para que el contagio sea menor (Montero-Medina, et al., 2020).

Sin embargo a provocado la reducción de sus horas laborales, es aquí donde sucede la problemática cuando una mujer pierde el trabajo se han dado situaciones donde terminan dependiendo de su pareja que a su vez se vuelve violenta, como es el caso de Ecuador un país que no cuenta con ingresos altos, lo que no permite que tengan una medida de protección por parte del gobierno porque muchas mujeres se dedican a trabajos informales, para ello se han adoptado las medidas necesarias para que exista atención y que las personas en este caso hombre o mujer que sean víctimas de violencia puedan continuar con sus trabajos labores sin ningún problema, también es importante la capacitación en los padres de familia para reconocer los diferentes tipos de violencia dentro del hogar y las afectaciones que provocan la vulneración de los derechos humanos. 


\section{CONCLUSIONES}

Se analizaron casos de violencia intrafamiliar y la realidad que tienen las familias ecuatorianas en tiempos de COVID-19, por cuanto se ha dado paso a que la víctima debe estar aislada junto con el agresor, esto les dificulta a las personas que sufren de violencia intrafamiliar específicamente a las mujeres, debido a que no puedan mantener el apoyo de sus familiares, amigos o de instituciones que les puedan brindar protección. Se identificó el tipo de violencia intrafamiliar que se ocasionó a la víctima, como una forma de maltrato realizado por una persona sobre otro integrante de la familia, estos actos de violencia pueden ser físicos, sexuales, psicológicos que pueden llegar a causar la muerte de la persona agredida (victima). Los resultados obtenidos a través del ECU911 demuestran que se han registrado un total de 8.196 llamadas por violencia intrafamiliar en el mes de marzo en donde se vulneran los derechos de la victimas ya que tienden a permanecer junto al agresor por más tiempo.

Se puede evidenciar que la pandemia por COVID-19, ha generado que varias familias pierdan el trabajo, dando así a que los estudiantes no puedan acceder a la educación porque no cuentan con los medios suficientes, debido a que sus padres no gozan de un trabajo estable y los problemas en el hogar son diariamente siendo más propensos a que sufran de violencia intrafamiliar.

En el Ecuador la violencia intrafamiliar ha estado presente más en el género femenino debido a que en esta situación de pandemia se está poniendo en riesgo la vida al convivir con el agresor, por esta razón las mujeres tienen la responsabilidad de atender y estar al pendiente de la salud de sus hijos todo esto ha generado violencia intrafamiliar en niveles muy altos, para ello es necesario informar a los miembros de la población acerca de los derechos que se han vulnerado para que exista una concientización. 
lustitia Socialis. Revista Arbitrada de Ciencias Jurídicas.

Año V. Vol. V. №3. Edición Especial. 2020-III:

Universidad Regional Autónoma de los Andes

Hecho el depósito de Ley: FA2016000064

ISSN: 2542-3371

FUNDACIÓN KOINONIA (F.K). Santa Ana de Coro, Venezuela

Laura Alicia Colcha-Ramos; Letty Elizabeth Córdova-Requena; Erika Guadalupe Salguero-Abarca

\section{FINANCIAMIENTO}

No monetario.

\section{AGRADECIMIENTO}

A la Universidad Regional Autónoma de Los Andes; por motivar el desarrollo de la Investigación.

\section{REFERENCIAS CONSULTADAS}

Chacón, C. (2020). 6.819 llamadas ha recibido el ECU-911 por violencia de género. [6,819 calls have received the ECU-911 for gender violence]. Recuperado de https://n9.cl/uvb4m

Cisneros-Trujillo, C. (2006). La violencia intrafamiliar: política criminal del Estado. [Domestic violence: criminal policy of the State]. Pontificia Universidad Javeriana. http://hdl.handle.net/10554/45092

Código Orgánico Integral Penal, COIP. Registro Oficial Suplemento 180 de 10-feb.-2014. Última modificación: 05-feb.-2018. Recuperado de https://n9.cl/xu3yj

De-Alencar-Rodrígues, R., \& Cantera, L. (2013). Intervención en violencia de género en la pareja: el papel de los recursos institucionales. [Intervention in intimate partner violence: the role of institutional resources]. Athenea Digital. Revista De Pensamiento E Investigación Social, 13(3), 75-100. https://doi.org/10.5565/rev/athenead/v13n3.1058

Guerra, P., V, D., \& Beltrán, D. (2020). La Desigualdad de las cargas laborales frente al COVID-19. [The Inequality of workloads against COVID-19]. CienciAméRica, 9(2), 244-250. http://dx.doi.org/10.33210/ca.v9i2.313

INEC. (2020). Los riesgos a los que se enfrentan las mujeres durante la emergencia sanitaria por el COVID-19 en Ecuador. [The risks women face during the health emergency due to COVID-19 in Ecuador]. Recuperado de https://n9.cl/v0ajl

López-Hernández, E., \& Rubio-Amores, D. (2020). Reflexiones sobre la violencia intrafamiliar y violencia de género durante emergencia por COVID-19. [Reflections on domestic violence and gender violence during the COVID-19 emergency]. CienciAméRica, 9(2),

312 321. http://dx.doi.org/10.33210/ca.v9i2.319 
Mayor-Walton, S, \& Salazar-Pérez, C. (2019). La violencia intrafamiliar. Un problema de salud actual. [Intrafamily violence. A current health problem]. Gaceta Médica Espirituana, 21(1), 96-105.

Metro Ecuador (2020). Denuncias por violencia contra la mujer y femicidios aumentó en Ecuador. [Complaints of violence against women and femicides increased in Ecuador]. Recuperado de https://n9.cl/3j2c

Ministerio de Agricultura y Agricultura (2019). Súper Mujer Rural, un pilar fundamental del desarrollo productivo nacional. [Super Rural Woman, a fundamental pillar of national productive development]. Recuperado de https://n9.cl/f9p2

Montero-Medina, D., Bolívar-Guayacundo, M., Aguirre-Encalada, L., \& MorenoEstupiñán, A. (2020). Violencia intrafamiliar en el marco de la emergencia sanitaria por el COVID-19. [Domestic violence in the framework of the health emergency caused by COVID-19]. CienciAméRica, 9(2), 261-267. http://dx.doi.org/10.33210/ca.v9i2.316

Organización Panameriana de la salud (2002). Informe mundial sobre la violencia y la salud: resumen. [World report on violence and health: summary]. Recuperado de https://n9.cl/vhhic

Puente-Martínez, A, Ubillos-Landa, S, Echeburúa, E, \& Páez-Rovira, D. (2016). Factores de riesgo asociados a la violencia sufrida por la mujer en la pareja: una revisión de meta-análisis y estudios recientes. [Risk factors associated with the violence against women in couples: a review of meta-analyzes and recent studies]. Anales de Psicología, 32(1), 295-306. https://dx.doi.org/10.6018/analesps.32.1.189161

Romero-Bello, D, \& González-Blanco, M. (2017). Violencia Doméstica según la percepción de la mujer. [Domestic Violence according to the perception of women]. Revista de Obstetricia y Ginecología de Venezuela, 77(1), 30-40.

Segura-Vera, M, \& Stranieri, M. (2016). Formación del personal de salud para el abordaje de la violencia de género en Venezuela. [Training of health personnel for addressing gender violence in Venezuela]. Comunidad y Salud, 14(2), 78-85.

Servicio Integrado de Seguridad ECU 911 (2020). ECU 911 superó los tres millones de llamadas recibidas durante la emergencia sanitaria. [ECU 911 exceeded three million calls received during the health emergency]. Recuperado de https://n9.cl/t9l2e 
lustitia Socialis. Revista Arbitrada de Ciencias Jurídicas.

Año V. Vol. V. №3. Edición Especial. 2020-III:

Universidad Regional Autónoma de los Andes

Hecho el depósito de Ley: FA2016000064

ISSN: 2542-3371

FUNDACIÓN KOINONIA (F.K). Santa Ana de Coro, Venezuela

Laura Alicia Colcha-Ramos; Letty Elizabeth Córdova-Requena; Erika Guadalupe Salguero-Abarca

Welland, J. (2017). Violence and the contemporary soldiering body. Security Dialogue, 48(6), 524-540. https://doi.org/10.1177/0967010617733355

(C2020 por los autores. Este artículo es de acceso abierto y distribuido según los términos y condiciones de la licencia Creative Commons Atribución-NoComercial-Compartirlgual 4.0 Internacional (CC BY-NC-SA 4.0)

(https://creativecommons.org/licenses/by-nc-sa/4.0/). 